\title{
On Topological Modifications of Newton's Law
}

\author{
E.G. Floratos ${ }^{a, b}$, G.K. Leontaris ${ }^{c}$ \\ ${ }^{a}$ Physics Department, University of Athens, Zografou 15784 Athens, Greece \\ ${ }^{b}$ Institute of Nuclear Physics, NCSR Demokritos, 15310, Athens, Greece \\ ${ }^{c}$ Theoretical Physics Division, Ioannina University, GR-45110 Ioannina, Greece
}

\begin{abstract}
Recent cosmological data for very large distances challenge the validity of the standard cosmological model. Motivated by the observed spatial flatness the accelerating expansion and the various anisotropies with preferred axes in the universe we examine the consequences of the simple hypothesis that the three-dimensional space has a global $\mathcal{R}^{2} \times \mathcal{S}^{1}$ topology. We take the radius of the compactification to be the observed cosmological scale beyond which the accelerated expansion starts. We derive the induced corrections to the Newton's gravitational potential and we find that for distances smaller than the $\mathcal{S}^{1}$ radius the leading $1 / r$-term is corrected by convergent power series of multipole form in the polar angle making explicit the induced anisotropy by the compactified third dimension. On the other hand, for distances larger than the compactification scale the asymptotic behavior of the potential exhibits a logarithmic dependence with exponentially small corrections. The change of Newton's force from $1 / r^{2}$ to $1 / r$ behavior implies a weakening of the deceleration for the expanding universe. Such topologies can also be created locally by standard Newtonian axially symmetric mass distributions with periodicity along the symmetry axis. In such cases we can use our results to obtain measurable modifications of Newtonian orbits for small distances and flat rotation spectra, for large distances at the galactic level.
\end{abstract}




\section{Introduction}

Nowadays there is accumulating evidence that the standard cosmological model at very large distances might not be fully consistent with the astronomical data [1]- 11]. Indeed, observations at very large scales point to the existence of a possible anisotropy in the global structure of the universe. Similarly, cosmological data for quasars indicate that the linear polarization angles of the emitted photons are correlated to a preferred axis at huge distances of the order of $1.5 \mathrm{Gpcs}$ [2]. In fact, there is evidence for a preferred cosmological axis when comparing accelerations and large peculiar galaxy velocities at different directions. Further, from CMB anisotropies observed at the lowest multipoles in the spherical harmonic decomposition of temperature, there is a hint of the existence of preferred axis 1 . The explanation of rotational curves of galaxies implies the possible existence of dark matter but eventually there are other explanations [9, 10]. The standard tests on the validity of Newton's law and general relativity gravitational corrections are confined inside the solar system, binary pulsars, and gravitational lensing.

The hypothesis that the Universe has a non-trivial topology and it is possibly multiconnected has been considered since the beginning of the 20th century [14] $]^{1}$. Further, similar ideas were developed afterwards [12] where a non-trivial topology was also assumed in considerations with regard to the quantum creation of the Universe and the origin of inflation [16, 17].

In the present note, we consider the possibility that matter does not only influence the surrounding space-time geometry but also the topology in various scales. This idea comes from the well known fact, that in order to describe local physics we need to select a complete set of smooth functions on the whole space in order to form delta functions, Greens functions etc, but then the topology of the space is crucial in determining this complete set. One possible and simple approach is to consider violations of Newton's law emerging from local changes of the topology of the Euclidean space $\mathcal{R}^{3}$ to $\mathcal{R}^{2} \times \mathcal{S}^{1}$. In a subsequent work we will develop further this idea in the framework of Einstein's Theory of General Relativity. At present, we will confine our analysis on the Newtonian limit of the above idea i.e. the global topology change of $\mathcal{R}^{3}$ Euclidean space to $\mathcal{R}^{2} \times \mathcal{S}^{1}$. We will obtain then, the exact solution for the Newton's potential interpolating between short distances where the usual $\frac{1}{r}$-behavior appears, to the large distance behavior $\log r$ for distances much larger the radius $R$ of $\mathcal{S}^{1}$. We will see that we obtain axial symmetric solutions with multipole terms and indications of correct qualitative behavior for the rotational spectra of galaxies. Other tests like gravitational lensing and spectra of CMB [7] require further studies.

We find also that our result is equivalent to that obtained from an infinite discrete and periodic mass distribution along the decompactified dimension. An analysis of these implications on the Keplerian orbits of Stars at distances where the first nonleading corrections are important, is also presented. A detailed comparison with the astrophysical data and the observed uncertainties in beyond the scope of this letter

\footnotetext{
${ }^{1}$ For recent surveys see for example [15].
} 
and will be the subject of a future publication. In the present note, in section 2 we derive and discuss in detail the properties of the $\mathcal{R}^{2} \times \mathcal{S}^{1}$ potential and in section 3 we analyse its implications on the trajectories of massive objects. The results are discussed in section 4.

\section{Gravitational Potential in $\mathcal{R}^{2} \times \mathcal{S}^{1}$}

Models for modifications of Newton's Gravity have already been proposed for large as well as for minuscule distances, the latter being only relevant in the presence of extra compact dimensions [21]. A survey of recent possibilities of modifications of the Newtonian Gravity can be found in [10, 11]2. Here, in this work we perform a mathematical analysis which will prove useful to examine whether in the absence of Dark Matter, such modifications could also be interpreted as a possible effect of the ordinary matter in the topology of the space.

We assume a three dimensional space with the topology $\mathcal{R}^{2} \times \mathcal{S}^{1}$. We parametrise the compact dimension of 'radius' $R$ as $z=R q$ with $q=[0,2 \pi)$. The Poisson equation for a point unit mass in this case is written

$$
\nabla_{2+1}^{2} \Phi=\delta^{(2)}\left(\vec{\tau}-\vec{\tau}_{0}\right) \frac{1}{R} \delta\left(q-q_{0}\right)
$$

with $\vec{\tau}=(x, y) \in \mathbb{R}^{2}$. Expanding in Fourier modes, the solution can be expressed as follows

$$
\Phi=-\frac{1}{(2 \pi)^{3} R} \sum_{m=-\infty}^{\infty} \int d^{2} k e^{i \vec{k} \cdot \vec{\tau}+\imath m q} \int_{0}^{\infty} d s e^{-s\left(k^{2}+\frac{m^{2}}{R^{2}}\right)}
$$

Performing the $\vec{k}$ Gaussian integration the potential takes the form

$$
\Phi=-\frac{1}{8 \pi^{2} R} \sum_{m=-\infty}^{\infty} e^{\imath m q} \int_{0}^{\infty} e^{-\frac{\tau^{2}}{4 s}-s \frac{m^{2}}{R^{2}}} \frac{d s}{s}
$$

To proceed with our computation, we split the infinite sum (3) into two parts

$$
\Phi=-\frac{1}{8 \pi^{2} R}\left[\int_{0}^{\infty} e^{-\frac{\tau^{2}}{4 s}} \frac{d s}{s}+2 \sum_{m=1}^{\infty} \cos m q \int_{0}^{\infty} e^{-\frac{\tau^{2}}{4 s}-s \frac{m^{2}}{R^{2}}} \frac{d s}{s}\right]=\Phi_{0}+\delta \Phi
$$

The first integral diverges, however, we observe that it is the result of the Fourier integration of the zero-mode

$$
\Phi_{0}=-\frac{1}{8 \pi^{2} R} \int d^{2} k e^{\imath \vec{k} \cdot \vec{\tau}} \int_{0}^{\infty} d s e^{-s k^{2}}=-\frac{1}{8 \pi^{2} R} \int d^{2} k \frac{e^{\imath \vec{k} \cdot \vec{\tau}}}{k^{2}}
$$

\footnotetext{
${ }^{2}$ For other types of modified gravity scenarios related to dark matter, the accelerating expansion of the universe etc see for example [18, 19, 20].
} 
This obeys the two-dimensional Poisson equation

$$
\nabla^{2} \Phi_{0}=\frac{1}{2 R} \delta^{(2)}(\vec{\tau})
$$

with the known solution

$$
\Phi_{0}=\frac{1}{4 \pi^{2}} \frac{1}{R} \ln \frac{\tau}{\tau_{0}}
$$

The result of the second integration in (4) is denoted with $\delta \Phi$ and it equals twice the modified Bessel function $2 K_{0}(m \tau / R)$. Hence, adding the two contributions the potential takes the form

$$
\Phi=\frac{1}{4 \pi^{2} R}\left(\ln \frac{\tau}{\tau_{0}}-2 \sum_{m=1}^{\infty} \cos (m q) K_{0}(m \tau / R)\right)
$$

We can obtain a closed form of the second term using a known formula from [22]. Plugging in the appropriate variables, we get

$$
\begin{aligned}
\sum_{m=1}^{\infty} K_{0}\left(m \frac{\tau}{R}\right) \cos m q & =\frac{1}{2}\left(\gamma+\ln \frac{\tau}{4 \pi R}\right)+\frac{\pi R}{2 \sqrt{\tau^{2}+(R q)^{2}}} \\
& +\frac{\pi}{2} \sum_{l=1}^{\infty}\left[\frac{R}{\sqrt{\tau^{2}+R^{2}(2 \pi l-q)^{2}}}-\frac{1}{2 \pi l}+\frac{R}{\sqrt{\tau^{2}+R^{2}(2 \pi l+q)^{2}}}-\frac{1}{2 \pi l}\right]
\end{aligned}
$$

Using this expansion we notice that the logarithms in (8) cancel each other. We may further choose the constant $\tau_{0}$ to be $\tau_{0}=4 \pi R e^{-\gamma} \approx 2.246 \pi R$ so the final form of the potential valid for all distances takes the form

$$
\begin{aligned}
\Phi & =-\frac{1}{4 \pi} \frac{1}{\sqrt{\tau^{2}+R^{2} q^{2}}} \\
& -\frac{1}{4 \pi} \sum_{l=1}^{\infty}\left[\frac{1}{\sqrt{\tau^{2}+R^{2}(2 \pi l-q)^{2}}}-\frac{1}{2 \pi l R}+\frac{1}{\sqrt{\tau^{2}+R^{2}(2 \pi l+q)^{2}}}-\frac{1}{2 \pi l R}\right]
\end{aligned}
$$

From this latter expression, we observe that the three dimensional Newton's potential is recovered in the limit $R \rightarrow \infty, q \rightarrow 0$ with $z=R q$ finite. We further observe that form of the potential in (9) can also be represented as the Newtonian potential of an infinite discrete mass distribution of period $2 \pi R$ along the decompactified dimension.

\subsection{The potential at short distances}

The above form of the potential encodes the axial symmetry of the topology of space and therefore it is interesting to investigate particular limiting cases with respect to the coordinate axes. However it is not easy to elaborate it further in its present form. 
In order to do this, we need to introduce a convenient parametrization. To this end, we define

$$
a_{l}=\frac{r}{2 \pi l R}
$$

and we note that $z=R q=r \cos \theta$. Now we can expand the square roots into Legendre polynomials for small $r /(2 \pi R)$

$$
\begin{aligned}
\frac{1}{\sqrt{\tau^{2}+R^{2}(2 \pi l \pm q)^{2}}} & =\frac{1}{2 \pi l R} \frac{1}{\sqrt{1+a_{l}^{2} \pm 2 a_{l} \cos \theta}} \\
& =\frac{1}{2 \pi l R} \sum_{k=0}^{\infty} P_{k}( \pm \cos \theta) a_{l}^{k}
\end{aligned}
$$

Substituting into the series form of $\Phi$ while using the property $P_{k}(-\cos \theta)=(-)^{k} P_{k}(\cos \theta)$ and changing the order of the two summations we obtain

$$
\begin{aligned}
\Phi & =-\frac{k}{4 \pi} \frac{1}{r}-\frac{k}{2 \pi} \sum_{l=1}^{\infty} \frac{1}{2 \pi l R}\left[\sum_{n=0}^{\infty} \frac{r^{2 n}}{(2 \pi l R)^{2 n}} P_{2 n}(\cos \theta)-1\right] \\
& =-\frac{k}{4 \pi} \frac{1}{r}\left(1+2 \sum_{n=1}^{\infty} \zeta(2 n+1)\left(\frac{r}{2 \pi R}\right)^{2 n+1} P_{2 n}(\cos \theta)\right)
\end{aligned}
$$

We remark first that the above expression for the potential holds for small distances $r /(2 \pi R)<1$ and moreover care should be taken in the interpretation of the spherical coordinate variables $r, \theta$ in order to implement the topology of the space $\mathcal{R}^{2} \times \mathcal{S}^{1}$.

In the following we are going to examine the behavior of the potential in various directions of $\mathcal{R}^{2} \times \mathcal{S}^{1}$ space. We study two cases for the potential, the first along the $z$-axis $(\theta=0)$ and the second on the $(x, y)$ plane $\left(\theta=\frac{\pi}{2}\right)$.

In the first case, $(\theta=0)$, we have $P_{n}(1)=1$ and the infinite sum in (12) can be expressed in terms of the Euler constant $\gamma$ and PolyGamma functions so that the potential along the $z$-axis is given in closed form

$$
V(r)=-\frac{k}{r}+\gamma+\frac{k}{\pi R}\left(\psi^{(0)}\left(1-\frac{r}{s}\right)+\psi^{(0)}\left(1+\frac{r}{s}\right)\right), s=2 \pi R
$$

For the second case $\theta=\frac{\pi}{2}$ and

$$
P_{2 n}(0)=\frac{(-1)^{n}(2 n) !}{2^{2 n}(n !)^{2}}
$$

The potential in this case takes the form

$$
V(r)=-\frac{k}{r}\left[1+2 \sum_{n=1}^{\infty} \frac{(-1)^{n}(2 n) ! \zeta(2 n+1)}{2^{2 n}(n !)^{2}}\left(\frac{r}{2 \pi R}\right)^{2 n+1}\right]
$$

For large $n$ the coefficients of the powers of the expansion parameter $s=r /(2 \pi R)$ goes as $(-1)^{n} / \sqrt{n}$, so we can approximate the above sum by appropriate combinations of the polylogarithmic functions $\mathrm{Li}_{\frac{1}{2}}$. The above considerations describe the behavior of the potential near the decompactification limit $r \rightarrow 0$. Figure 1 shows the modification of the gravitational potential for $\mathcal{R}^{2} \times \mathcal{S}^{1}$ topology in comparison to $\mathcal{R}^{3}$ case. 


\subsection{The potential for large distances}

Because of the compactification radius bound, large distances where $r /(2 \pi R)>1$, can be reached only in the direction $\theta=\frac{\pi}{2}$ or $q=0$. In this case, expanding the Bessel function in (8) for large values of $t=m \rho$ with $\rho=\tau / R$ we have

$$
\begin{aligned}
K_{0}(t) & =\sqrt{\frac{\pi}{2 t}} e^{-t} \sum_{n=0}^{\infty} \frac{[(2 n-1) ! !]^{2}}{n !} \frac{1}{(-8 t)^{n}} \\
& =\sqrt{\frac{\pi}{2 t}} e^{-t}\left(1-\frac{1}{8 t}+\frac{3^{2}}{2 !(8 t)^{2}}-\frac{(3 \cdot 5)^{2}}{3 !} \frac{1}{(8 t)^{3}}+\frac{(3 \cdot 5 \cdot 7)^{2}}{4 !} \frac{1}{(8 t)^{4}}+\cdots\right)
\end{aligned}
$$

and performing the sum in (8) we obtain

$$
\begin{aligned}
\sum_{m=1}^{\infty} K_{0}(m \rho) & =\sqrt{4 \pi} \sum_{n=0}^{\infty} \frac{[(2 n-1) ! !]^{2}}{n !} \frac{(-1)^{n}}{(8 \rho)^{n+\frac{1}{2}}} \operatorname{Li}_{n+\frac{1}{2}}\left(e^{-\rho}\right) \\
& =\sqrt{\frac{\pi}{2 \rho}}\left(\operatorname{Li}_{\frac{1}{2}}\left(e^{-\rho}\right)-\frac{1}{8 \rho} \operatorname{Li}_{\frac{3}{2}}\left(e^{-\rho}\right)+\frac{9}{128 \rho^{2}} \operatorname{Li}_{\frac{5}{2}}\left(e^{-\rho}\right)+\cdots\right)
\end{aligned}
$$

The complete potential is obtained by adding the logarithmic term. This in the large $\tau / R$ regime becomes

$$
V_{c}(\rho)=\frac{1}{2 \pi^{2}} \frac{1}{R}\left(-C+\frac{1}{2} \log (\rho)-\sqrt{\frac{\pi}{2 \rho}}\left(\operatorname{Li}_{\frac{1}{2}}\left(e^{-\rho}\right)-\frac{1}{8 \rho} \operatorname{Li}_{\frac{3}{2}}\left(e^{-\rho}\right)+\cdots\right)\right)
$$

where $C$ is the constant

$$
C=\ln (2 \sqrt{\pi})-\gamma / 2
$$

We note here that the corrections of the logarithmic term are exponentially small. In figure 2 we plot the effective potential $V_{\text {eff }}(\tau)=V_{c}+\ell^{2} /\left(2 m \tau^{2}\right)$, taking three values for $\ell /(2 m)=0.06,0.09,0.12$ where $\ell$ is the conserved angular momentum and $m$ the mass. For large values of $\tau / R$ we add correction terms $\operatorname{Li}_{n+\frac{1}{2}}$ up to $n=2$. These correspond to the right piece of the curves (red color). At distances around $r \approx 2 \pi R$
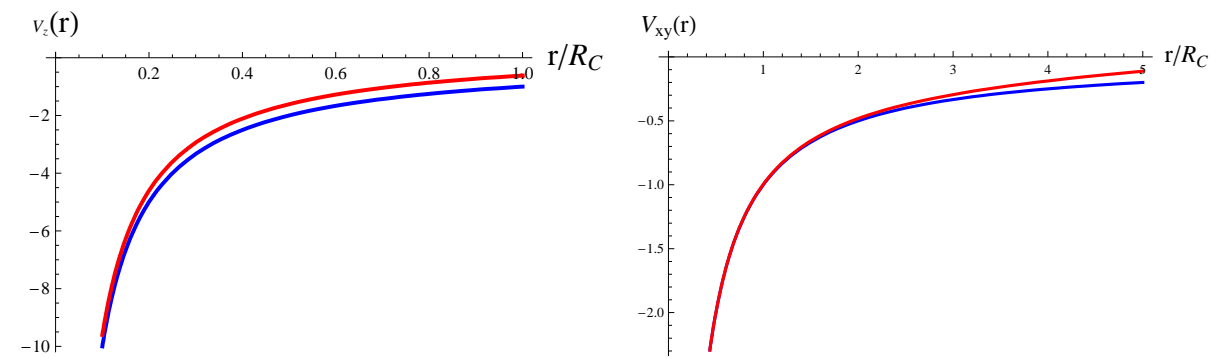

Figure 1: Modifications of the Gravitational potential (in arbitrary units) a) along the $z$-axis b) along the $x y$-plane. Blue (lower) lines corresponds to $\mathcal{R}^{3}$-case, the red ones (upper) to $\mathcal{R}^{2} \times \mathcal{S}^{1}$ topology. 
the curves cross the abscissa and for larger $r$-values, the potential becomes positive and increasing. Clearly this is due to the logarithmic dependence of the potential. To plot the potential for small $\tau / R$ we use the formula (12) taking $n=10$ terms of the sum (in practise only few of them suffice). These values are represented by the left part of the curves (blue color). We observe that the two curves match perfectly around the area $\rho \approx 1$, thus our approximation is valid for small $\tau / R \lesssim 1$ as well as large $\tau / R \gtrsim 1$ values.

We observe now that the corrections terms constitute an alternating series of polylogarithmic functions of the exponential $e^{-\rho}$. Thus increasing the values $\rho>1$, their value decreases and the dominant contribution comes from the logarithmic term. So, for large distances the rotational spectra of massive objects are flat

$$
v \approx \text { constant }
$$

We notice here that axially symmetric periodic Newtonian mass distributions along the $z$-direction can mimic the effect of the compactification $\mathcal{R}^{2} \times \mathcal{S}^{1}$. So although presently there is no observational basis for such mass distributions, ( for example concentration of galaxies along cosmic strings) we can choose the compactification radius $R$ to be of the order of the average intergalactic scale. This way, our mechanism could mimic the effects of the dark matter.

\section{Modification of the orbits}

The modifications of the potential discussed above, are expected to be negligible at small distances. Indeed, one can notice that the relative coefficient of the first correction term is $\frac{2 \zeta(3)}{(2 \pi)^{3}} P_{2}(0) \approx-0.005$, thus for $r \ll R$ its contribution diminishes. There are

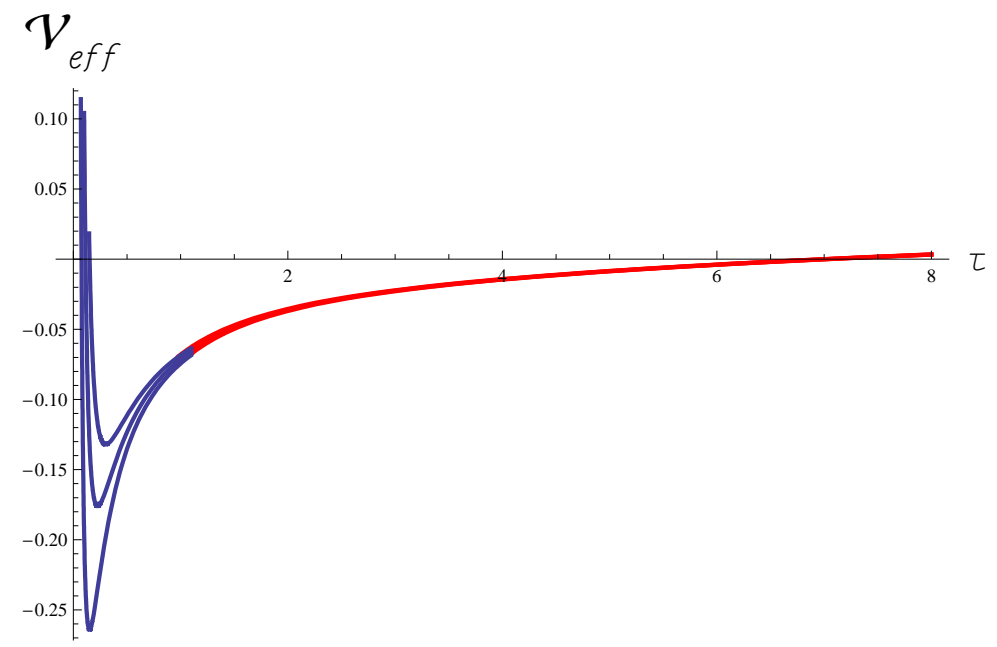

Figure 2: Plot of the approximate formulae of $\mathcal{R}^{2} \times \mathcal{S}^{1}$ gravitational potential $V_{\text {eff }}(\tau / R)$ for small $\tau / R$ ( left blue curves) and large $\tau / R$ (right red curves). 
considerable deviations from Newton's mechanics however, when dealing with distances at very large scales. In this case the relation (8) gives the leading logarithmic behavior with exponentially small corrections.

At intermediate distances the effect of the corrections will be important but we need to know the exact behavior of the potential which, although complicated algebraically, numerically interpolates nicely between the $1 / \tau$ and $\log (\tau)$ behavior. Here we examine the effects of the violations of the Newton's law in the $\mathbb{R}^{2}$-plane for the orbits of massive objects and for small distances.

For distances inside the compactification scale, where the first non-leading correction to the Newtonian law plays a role it is interesting to consider the nature of modifications on the orbits of massive objects. We start with the calculation of the force taking into account the first non-leading term and in polar coordinates $(r, \theta, \phi)$ we find

$$
\vec{F}=\vec{F}_{0}+\vec{F}_{c}=k\left\{-\frac{1}{r^{2}}+\frac{3 r \zeta(3)}{8 \pi^{3} R^{3}}\left(\cos 2 \theta+\frac{1}{3}\right),-\frac{3 r \zeta(3)}{8 \pi^{3} R^{3}} \sin 2 \theta, 0\right\}
$$

The form of the correction to Newton's force is similar to the tidal forces on a dipole. At this level of approximation we observe that the axially symmetric additional forces are small and repulsive for $\cos (2 \theta)>-\frac{1}{3}$ and attractive otherwise. For the study of the effect of this correction to the orbits we restrict our attention to the planar motion $\left(\theta=\frac{\pi}{2}\right), F_{\theta}=0$ while

$$
F_{r} \approx-\frac{k}{r^{2}}-\frac{k \zeta(3)}{4 \pi^{3} R^{3}} r
$$

The corresponding equation of motion is

$$
\frac{d^{2} u}{d \varphi^{2}}+u=\frac{k m}{\ell^{2}}\left(1+\frac{2 \zeta(3)}{(2 \pi R)^{3}} \frac{1}{u^{3}}\right)
$$

where $u=\frac{1}{r}$. This equation is easily solved using the conservation of the angular momentum,

$$
\varphi-\varphi_{0}=\int_{r_{0}}^{r} \frac{d \tau}{\tau^{2} \sqrt{\frac{2 m}{\ell^{2}}\left(\mathcal{E}-V(\tau)-\frac{\ell^{2}}{2 m \tau^{2}}\right)}}
$$

where

$$
\ell=m r^{2} \dot{\varphi}
$$

The correction to the Newton's potential is an even-power series of $r$, the most dominant being the harmonic term. Thus, the effective potential can be approximated by

$$
V_{e f f}(r)=-\frac{k}{r}\left(1-\frac{\ell^{2}}{2 m k r}-\delta \frac{\zeta(3)}{(2 \pi)^{3}}\left(\frac{r}{R}\right)^{3}\right)
$$


where $\delta=0$ for the classical Newton's law and $\delta=1$ for the corrected potential. The exact computation of the integral would introduce complicated elliptic functions, however, since the correction is small it can be treated perturbatively by expanding appropriately the denominator and computing the relevant integrals. Instead of this, we will work out in the next section the corresponding equation of motion.

\subsection{The equation of motion}

In the approximation discussed above we may analyse easily the motion using the standard approach by deriving the differential equation for the new variable $u=1 / r$ introduced above. The perturbed differential equation is written as follows

$$
\frac{d^{2} u}{d \varphi^{2}}+u=\alpha\left(1+\epsilon u^{-3}\right)
$$

where $\alpha=k m / \ell^{2}$ and the parameter $\epsilon$ can be deduced from (20)

$$
\epsilon=\frac{\zeta(3)}{4 \pi^{3} R^{3}}
$$

We assume solutions of the form

$$
u=u_{0}+\epsilon u_{1}
$$

where $u_{0}$ is the classical elliptic solution

$$
r_{0}(\varphi)=\frac{a\left(1-e^{2}\right)}{1+e \cos (\varphi)}=\frac{1}{u_{0}(\varphi)}
$$

where as usually, a,e stand for the semimajor axis and the eccentricity 3 . We seek solutions for the perturbed equation which have the same angular momentum as the unperturbed one, thus $\delta \ell=0$. Substituting into (24) and using the equation (26) for $u_{0}(\varphi)$, one finds

$$
\frac{d^{2} u_{1}}{d \varphi^{2}}+u_{1}=\frac{\alpha}{u_{0}^{3}(\varphi)}
$$

This has the solution

$$
u_{1}(\varphi)=\frac{I(\varphi)}{\alpha^{2}}=\frac{\cos \varphi I_{s}(\varphi)-\sin \varphi I_{c}(\varphi)}{\alpha^{2}}
$$

where $I_{s}(\varphi)$ and $I_{c}(\varphi)$ are given by

$$
\begin{aligned}
& I_{c}(\varphi)=\frac{3 e}{\left(1-e^{2}\right)^{5 / 2}} \tan ^{-1}\left(\sqrt{\frac{1-e}{1+e}} \tan \frac{\varphi}{2}\right)-\frac{\left(2+e^{2}+\left(1+2 e^{2}\right) e \cos \varphi\right) \sin \varphi}{2\left(1-e^{2}\right)^{2}(1+e \cos \varphi)^{2}} \\
& I_{s}(\varphi)=\frac{1}{2 e}\left(\frac{1}{(1+e)^{2}}-\frac{1}{(1+e \cos \varphi)^{2}}\right)
\end{aligned}
$$

\footnotetext{
${ }^{3}$ These are defined as $a=-\frac{k}{2 E}$ and $e=\sqrt{1-\frac{2 E \ell^{2}}{m k^{2}}}=\sqrt{1-\frac{\ell^{2}}{k m a}}$. Note also that the parameter $\alpha$ is related to the physical parameters $a, e$ by $\alpha^{-1}=a\left(1-e^{2}\right)$.
} 


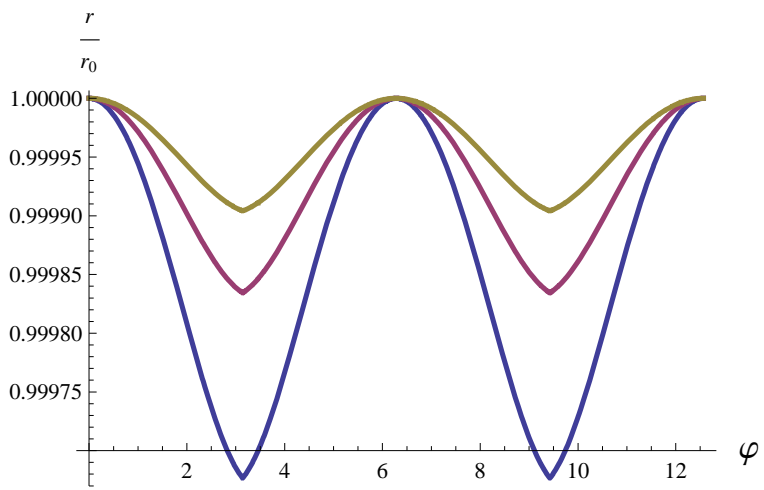

Figure 3: The ratio $\frac{r(\varphi)}{r_{0}(\varphi)}$ with $r$ being the distorted orbit in the presence of the correction term in $\mathcal{R}^{2} \times \mathcal{S}^{1}$ topology. The three curves (upper, middle, lower) correspond to three different ratios $a / R=1 / 6,1 / 5,1 / 4$ where $a$ is the semimajor axis of the unperturbed orbit and $R$ the $\mathcal{S}^{1}$-radius.

To get a feeling of the corrections implied on the trajectories of physical objects we form the ratio of the corrected over the unperturbed solution. To this end, we expand the ratio in terms of the dimensionless quantity $\epsilon / \alpha^{3}$ and find that for small eccentricities $e \ll 1$ the essential modification of the orbits is captured by the formula

$$
r(\varphi) \approx r_{0}(\varphi)\left(1-\xi^{3} \sin ^{2} \frac{\varphi}{2}\right)
$$

where $r_{0}(\varphi)$ is given by (26) and we have defined

$$
\xi=\left(\frac{\zeta(3)}{2}\right)^{\frac{1}{3}} \frac{a}{\pi R}
$$

For $R$ of the order of galactic distances and $a$ a typical solar system distance, this implies a negligible distortion on the orbits.

Next we study the impact on the trajectories of massive objects with bounded motion, using three values for the ratio $\lambda=1 / 6,1 / 5,1 / 4$ while assuming the eccentricity $e=0.068$. For an assumed massive object subject to the forces due to a potential generated at the origin, the corresponding ratios are plotted in figure (3). It is obvious that for massive objects at the boundaries of galaxies the radius $R$ has to be taken of the same order to have an observable modification of Newtonian orbits.

We close our analysis with the following remark. Suppose that in the absence of the $\mathcal{R}^{2} \times \mathcal{S}^{1}$-effects the orbit of an object with eccentricity $e$ is determined as usual by $r_{0}(\varphi)$ given by (26). Substituting, the solution (29) can be also written as follows

$$
r(\varphi) \approx a(1-e) \frac{1-\xi^{3} \sin ^{2} \frac{\varphi}{2}}{1-\frac{2 e}{1+e} \sin ^{2} \frac{\varphi}{2}}
$$


For objects satisfying $\xi^{3}=2 e /(1+e)$, or equivalently

$$
a=\pi R\left(\frac{1}{\zeta(3)} \frac{4 e}{1+e}\right)^{\frac{1}{3}}
$$

and to first order approximation on the eccentricity $e$, we obtain

$$
r(\phi)=a(1-e)=\mathrm{constant}
$$

Therefore, certain "would be" elliptic orbits in $\mathcal{R}^{3}$ space, in the case of $\mathcal{R}^{2} \times \mathcal{S}^{1}$ topology appear to be approximately circular.

\section{Discussion and conclusions}

Motivated by the fact that several recent astronomical and cosmological observations are challenging the theory of General Relativity at very large distances, due to accelerated expansion, axial anisotropies, planarity of CMB multipoles, dark energy etc in this note we have considered the role of a non-trivial topology of the universe in the Newtonian approximation without invoking extra dimensions.

Assuming the experimentally determined flatness of the spatial universe, we test at a qualitative level the proposal of the simplest periodic multiverse along the third spatial dimension or equivalently the assumption of a spatial topology $\mathcal{R}^{2} \times \mathcal{S}^{1}$ with a radius of the compactified dimension of the order of the distance where the acceleration starts, $L=2 \pi R$.

Within the aforementioned topology of the space, we have determined the exact Newtonian potential at any distance $r$.

For small $r / R$ ratios we find the corrections to the three dimensional Newton's $1 / r$ potential to be of the form of tidal forces $(r / R)^{n} P_{n}(\cos \theta)$, with an anisotropy axis along the third dimension. Another interesting finding at these distances is the repulsive accelerated motion along the axial direction in consistency with the observed large peculiar velocities of galaxies along the observed axes at some statistical significance level.

On the other hand, for large distances, where $r / R>1$, we get a smooth transition of the potential to the two dimensional one, $\log (r)$, with exponentially small corrections. This has the immediate consequence that for distances larger than $L$ we obtain smaller decelerations due to the appearance of the two dimensional attractive force $1 / r$, instead of the $1 / r^{2}$ of three dimensions, thus a relative acceleration.

Moreover, we expect that for such large distances or angular separations there must be a flattening of the CMB multipoles due to lower dimensionality of space.

Choosing smaller scales for the compactification radius could be afforded only in some General Relativity model, where the compactification is local and not global. Another possibility is to mimic the compactification of the third dimension with spatial axially symmetric and periodic in the third direction Newtonian mass distributions. In such a patch -say around a galaxy- we obtain that the trajectories of massive stars 
at the boundaries of the galaxy have flat rotational spectra and small but measurable perturbations of the periodic orbits for smaller distances.

We find the above results encouraging and the proposed topological modification of gravity worth of extending, in a future work, in the framework of General Relativity.

Acknowledgements We would like to thank CERN Theory Division for kind hospitality where part of this work has been done. The research Project is co-financed by the European Union - European Social Fund (ESF) \& National Sources, in the framework of the program "THALIS" of the "Operational Program Education and Lifelong Learning" of the National Strategic Reference Framework (NSRF) 2007-2013. 


\section{References}

[1] M. Tegmark, A. de Oliveira-Costa and A. Hamilton, Phys. Rev. D 68 (2003) 123523 arXiv:astro-ph/0302496.

K. Land and J. Magueijo, Phys. Rev. Lett. $95 \quad$ (2005) 071301 arXiv:astro-ph/0502237.

[2] D. Hutsemekers, R. Cabanac, H. Lamy and D. Sluse, Astron. Astrophys. 441, 915 (2005) arXiv:astro-ph/0507274.

[3] A. Kashlinsky, F. Atrio-Barandela, D. Kocevski and H. Ebeling, results and cosmological implications," Astrophys. J. 686 (2009) L49 [arXiv:0809.3734 [astro-ph]].

[4] C. H. Lineweaver, L. Tenorio, G. F. Smoot, P. Keegstra, A. J. Banday and P. Lubin, Astrophys. J. 470 (1996) 38 arXiv:astro-ph/9601151.

[5] C. J. Copi, D. Huterer, D. J. Schwarz and G. D. Starkman, Adv. Astron. 2010 (2010) 847541 arXiv:1004.5602 [astro-ph.CO]].

[6] I. Antoniou and L. Perivolaropoulos, Comparison with Other Probes," JCAP 1012, 012 (2010) arXiv:1007.4347 [astro-ph.CO]].

J. Grande and L. Perivolaropoulos, Phys. Rev. D $84 \quad$ (2011) 023514 arXiv:1103.4143 [astro-ph.CO]].

[7] F. R. Urban, A. R. Zhitnitsky, arXiv:1011.2425 [astro-ph.CO]].

[8] R. Watkins, H. A. Feldman and M. J. Hudson, Standard LCDM Cosmology," Mon. Not. Roy. Astron. Soc. 392 (2009) 743 [arXiv:0809.4041 [astro-ph]].

[9] M. Milgrom, Astrophys. J. 270 (1983) 384.

J. Bekenstein and M. Milgrom, Astrophys. J. 286 (1984) 7.

[10] R. H. Sanders and S. S. McGaugh, Ann. Rev. Astron. Astrophys. 40 (2002) 263 arXiv:astro-ph/0204521.

[11] T. Clifton, P. G. Ferreira, A. Padilla, C. Skordis, [arXiv:1106.2476 [astro-ph.CO]].

[12] Y. B. Zeldovich and A. A. Starobinsky, Sov. Astron. Lett. 10 (1984) 135.

Y. B. Zeldovich and A. A. Starobinsky, Sov. Phys. JETP 34 (1972) 1159 [Zh. Eksp. Teor. Fiz. 61 (1971) 2161].

N. J. Cornish, D. N. Spergel and G. D. Starkman, Class. Quant. Grav. 15 (1998) 2657 astro-ph/9801212.

J. Barrow and H. Kodama, Class. Quant. Grav. 18 (2001) 1753 gr-qc/0012075].

G. Aslanyan and A. V. Manohar, arXiv:1104.0015 [astro-ph.CO]. 
[13] V. G. Gurzadyan and R. Penrose, arXiv:1104.5675 [astro-ph.CO]. Yu. P. Goncharov and A. A. Bytsenko, Phys. Lett. B 160 (1985) 385.

A. D. Linde, JCAP 0410 (2004) 004 [arXiv:hep-th/0408164].

[14] W. de Sitter, 1917a, Mon. Not. R. Astron. Sot., 78, 3 (also 1916, 76, p. 49; 77, p. 155)

A. Friedmann, 1924, Z. Phys. 21, 326

G. Lemaitre, 1958, in La Structure et 1'Evolution de l'univers, Onzit me Conseil de Physique Solvay. R. Stoops, ed. Brussels : Stoops, pp.l-25.

[15] M. Lachieze-Rey, J. -P. Luminet, Phys. Rept. 254, 135-214 (1995). gr-qc/9605010. J. -P. Luminet, J. Weeks, A. Riazuelo, R. Lehoucq and J. -P. Uzan, Nature 425 (2003) 593 astro-ph/0310253.

E. Gausmann, R. Lehoucq, J. -P. Luminet, J. -P. Uzan and J. Weeks, Class. Quant. Grav. 18 (2001) 5155 gr-qc/0106033.

[16] A. Vilenkin, Phys. Lett. B117, 25 (1982).

[17] D. A. Easson, P. H. Frampton and G. F. Smoot, Phys. Lett. B 696 (2011) 273 arXiv:1002.4278 [hep-th]].

[18] S. 'i. Nojiri and S. D. Odintsov, eConf C 0602061 (2006) 06 [Int. J. Geom. Meth. Mod. Phys. 4 (2007) 115] [hep-th/0601213].

[19] E. J. Copeland, M. Sami and S. Tsujikawa, Int. J. Mod. Phys. D 15, 1753 (2006) hep-th/0603057.

[20] R. P. Woodard, Lect. Notes Phys. 720 (2007) 403 astro-ph/0601672.

[21] E. G. Floratos and G. K. Leontaris, Phys. Lett. B 465 (1999) 95 arXiv:hep-ph/9906238.

A. Kehagias and K. Sfetsos, Phys. Lett. B 472 (2000) 39 arXiv:hep-ph/9905417]. E. G. Floratos, G. K. Leontaris and N. D. Vlachos, Phys. Lett. B 694 (2011) 410 arXiv:1008.0765 [hep-ph]].

[22] I.S. Gradshteyn and I.M. Ryzhik, "Table of Integrals, Series, and Products" formula 8.526(1); Seventh Edition, 2007. 